\title{
Reduzierung der Verletzlichkeit durch internationale Kooperation
}

\author{
Gerade Regionen, die besonders von den Folgen \\ des Klimawandels betroffen sein werden, sind \\ häufig durch Armut und politisch schwierige \\ Rahmenbedingungen geprägt. Internationale \\ Zusammenarbeit bei der Anpassungsforschung \\ können die Fähigkeiten zur Bewältigung dieser \\ Herausforderungen erheblich stärken. \\ Von Esther Chrischilles
}

D er Klimaschutz hat mittlerweile eine Zielmarke. Auf dem UN-Klimagipfel von Cancún im Dezember 2010 verständigte sich die internationale Staatengemeinschaft darauf, den globalen Temperaturanstieg auf zwei Grad Celsius zu begrenzen. Was auf den ersten Blick wie die stillschweigende Akzeptanz des Klimawandels klingt, ist tatsächlich ein ambitioniertes Ziel. Zudem ist bereits eine globale Erderwärmung um zwei Grad Celsius ist mit erheblichen Risiken für ökologische, soziale und ökonomische Systeme verbunden. Meeresspiegelanstiege, veränderte Erwärmungs- und Niederschlagsmuster und häufiger auftretende Wetterextreme werden Länder und Regionen weltweit vor große Herausforderungen stellen (IPCC 2008).

\section{Das Zwei-Grad-Ziel}

Mit der wissenschaftlichen Evidenz zu klimatischen Veränderungen hat politisch wie wissenschaftlich eine stärkere Hinwendung zur Strategie der Anpassung an den Klimawandel stattgefunden. Schon im ersten Sachstandsbericht des Intergovernmental Panel on Climate Change (IPCC) von 1990 wird Anpassung an den Klimawandel als integrale strategische Handlungsoption empfohlen, wobei Anpassung als „Initiativen und Maßnahmen, um die Empfindlichkeit natürlicher und menschlicher Systeme gegenüber tatsächlichen oder erwarteten Auswirkungen der Klimaänderung zu verringern“ verstanden werden (IPCC 2008, S.86). Auch wenn Klimaanpassung in Bezug auf den Klimawandel als komplementäre Strategieoption zum Klimaschutz zu betrachten ist, wird das Thema in der Klimawandelforschung mittlerweile durchaus eigenständig behandelt (Chrischilles/Mahammadzadeh 2011).

Auch die internationale Staatengemeinschaft der UN-Klimarahmenkonvention hat Anpassungserfordernisse auf ihren letzten Gipfeltreffen immer deutlicher adressiert. Ein wesentlicher Treiber sind dabei vor allem Entwicklungsländer und kleinere Inselstaaten, in denen Folgen des Klimawandels schneller und deutlicher wirksam sein werden. Auf dem Klimagipfel von Kopenhagen hat sich die Staatengemeinschaft einmal mehr zu besserer Zusammenarbeit bei der Klimaanpassung bekannt, um die klimawandelbedingten Verletzlichkeiten in Entwicklungsländern zu reduzieren (FCCC/SBI/2009/L27 2009). Dazu gilt es jedoch zunächst einmal Klarheit über die Bedeutung des Begriffes Verletzlichkeit, wie er in der internationalen Anpassungsdebatte wie selbstverständlich verwendet wird, herzustellen.

\section{Verletzlichkeiten gegenüber dem Klimawandel}

Verletzlichkeit oder auch Vulnerabilität wird im Kontext des Klimawandels häufig als Analysemodell verwendet, um mögliche Schadensrisiken in Mensch-Umweltsystemen zu beschreiben, die durch ein externes klimawandelbedingtes Ereignis hervorgerufen werden (Dietz 2006). Darauf aufbauend können geeignete Handlungsoptionen zur Anpassung dieser Systeme formuliert werden.

Eine Analyse von klimawandelbedingten Verletzlichkeiten beinhaltet in der Regel die Komponenten Sensitivität, Exposition und Anpassungskapazität. Anpassung zielt vor allem darauf, die Senistivität menschlicher und natürlicher Systeme zu reduzieren. Sensitivität oder auch Anfälligkeit beschreibt „den Grad, zu dem ein System durch Klimavariabilität oder Klimaveränderung beeinflusst wird, sei es negativ oder positiv" (IPCC 2008, S. 96). Die Sensitivität eines Systems, oder auch räumlich gesehen einer Region, ergibt sich vor allem aus verschiedenen regionalen Gegebenheiten beziehungsweise den Eigenschaften der regionalen ökonomischen, sozialen oder biologischen Systeme. Beispielsweise ist eine Küstenregion, die sehr dicht besiedelt ist, anfälliger gegenüber einer erwarteten Sturmflut, als ein unbewohntes Gebiet. Es entstehen höhere negative Folgen, beispielsweise in Form von Personen- und Sachschäden. Wird also das erwartete Klimaereignis (Exposition) ins Verhältnis zur regionalen Anfälligkeit (Sensitivität) gesetzt, ergibt sich die negative Betroffenheit einer Region. Wenn Klimaveränderungen direkten oder indirekten Einfluss auf wesentliche Funktionen nehmen, ist die Region besonders negativ betroffen.

Im ersten Schritt einer Vulnerabilitätsanalyse muss danach gefragt werden, inwiefern und wie stark eine Region negativ durch direkte oder indirekte Klimafolgen betroffen ist (Chrischilles/Mahammadzadeh 2011). Negative Betroffenheit bezeichnet die potenziell entstehenden Klimafolgen, ohne dabei die Fähigkeiten, Ressourcen und Institutionen eines Landes oder einer Region zu berücksichtigen, mit denen wirksame Maßnah- $\rightarrow$ 
men zur Anpassung umgesetzt werden können. Die Fähigkeiten zur Anpassung an Klimafolgen werden als Anpassungskapazität bezeichnet (IPCC 2008). Je größer die Anpassungskapazität ist, desto besser kann eine Region ihren Grad der Anpassung erhöhen und so ihre Sensitivität reduzieren (1). Unter Berücksichtigung der Anpassungskapazitäten ergibt sich die eigentliche Vulnerabilität einer Region (Zebisch/Grothmann 2005). Eine Küstenregion beispielsweise wäre zwar von einer Sturmflut negativ betroffen, sie ist jedoch kaum vulnerabel, wenn sie personelle, technische und finanzielle Ressourcen für einen adäquaten Küstenschutz besitzt. Regionen mit einer hohen Anpassungskapazität sind besser in der Lage, auf potenzielle Gefahren zu reagieren und sich darauf vorzubereiten. Anpassungskapazität ist dabei nicht gleichzusetzen mit Anpassungsmaßnahmen. Unter Anpassungskapazität sind vielmehr die Voraussetzungen für solche Maßnahmen zu verstehen, beispielsweise der Zugang zu entsprechenden Technologien, Know-how oder institutionelle Arrangements. Die Sensitivität eines Systems kann nur durch die tatsächliche Nutzung dieser Kapazitäten reduziert werden.

Neagtive Betroffenheit beschreibt sozusagen den Status quo einer Risikoanalyse und sagt etwas über das potenzielle Schadensrisiko bei einer klimatischen Veränderung aus. Verletzlichkeit hingegen berücksichtigt die Möglichkeiten, sich zeitnah auf den Klimawandel vorzubereiten. Sie beschreibt das Schadensausmaß, das eintreten würde, wenn alle vorhandenen Kapazitäten zuvor genutzt würden, um den Anpassungsgrad zu erhöhen.

Verletzlichkeit ist folglich kein statischer Zustand, sondern über drei Strategien beeinflussbar:

- Maßnahmen zur Treibhausgasreduktion können das Ausmaß des Klimawandels und seiner Folgen reduzieren. Die Reduktion der Vulnerabilität erfolgt durch die Vermeidung beziehungsweise Verringerung der Exposition.

I Indem Anpassungskapazitäten genutzt und Anpassungs-

Abbildung 1: Elemente zur Bestimmung der Vulnerabilität

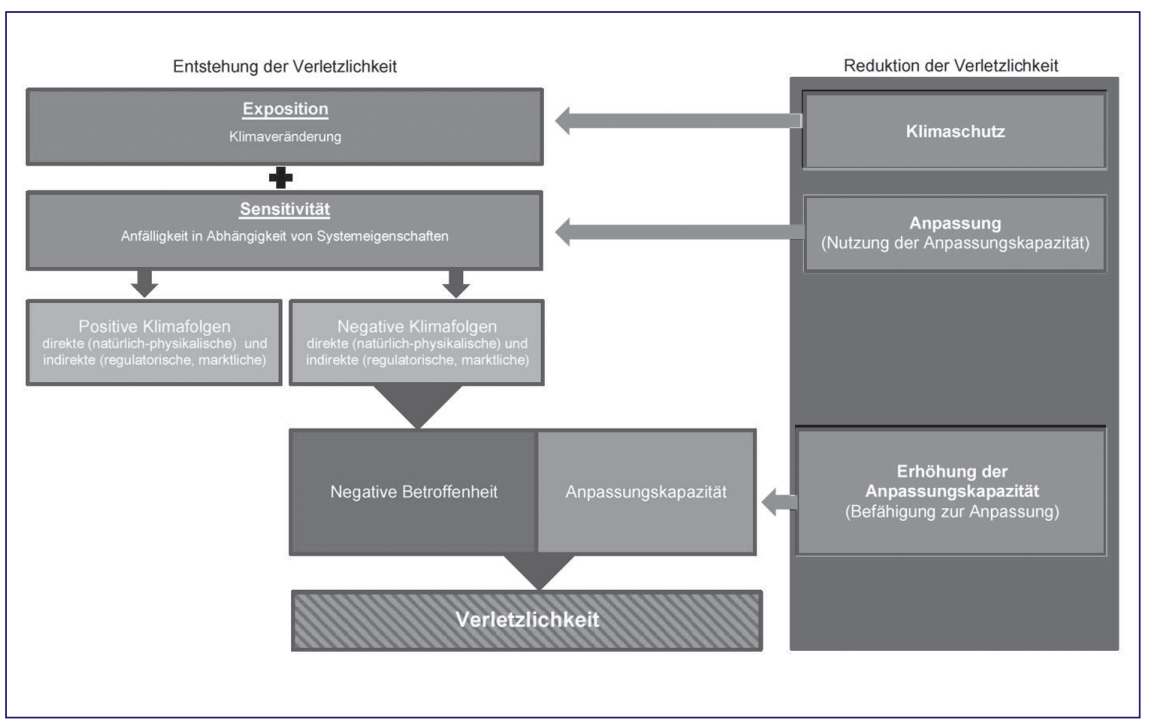

Quelle: Eigene Darstellung maßnahmen durchgeführt werden, kann die Anfälligkeit der regionalen Systeme reduziert werden. Die Reduktion der Vulnerabilität erfolgt durch Verringerung der Sensitivität.

I Indem die Fähigkeiten zur Anpassung gezielt gestärkt werden, kann sich eine negativ betroffene Region besser vorbereiten. Durch eine Erhöhung der Anpassungskapazität wird die Verletzlichkeit reduziert. Damit sind noch keine Anpassungsmaßnahmen verbunden, vielmehr geht es um die Befähigung zur Anpassung.

Zur Erhöhung der Anpassungskapazität zählt auch der verbesserte Zugang zu Wissen über die Folgen des Klimawandels und Möglichkeiten zur Anpassung. Die Kooperation in der internationalen Anpassungsforschung und Ergebnistransfer spielt in diesem Zusammenhang eine bedeutende Rolle.

\section{Globale Verteilung der Verletzlichkeit}

Klimawandel vollzieht sich lokal unterschiedlich, verursacht demnach ungleiche Expositionen. Die Folgen des Klimawandels treffen dabei auf differierende Systemeigenschaften, eine ungleiche Sensitivität sowie unterschiedliche Anpassungskapazitäten. Die drei Determinanten verdeutlichen, warum Verletzlichkeiten sich regional äußerst heterogen darstellen. Nationale Forschungsprogramme zur Anpassung setzen daher häufig auf kleinräumiger beziehungsweise regionaler Ebene an. Auf internationaler Ebene divergieren die Auswirkungen des Klimawandels noch stärker und werden besonders in solchen Ländern wirksam, die vergleichsweise anfällig sind. Entwicklungsländer beispielsweise sind häufig durch extreme Klimabedingungen, wie heißes und trockenes Klima oder starke jahreszeitliche Schwankungen gekennzeichnet. Auch extreme Wetterereignisse wie Wirbelstürme oder Hochwasser treten dort auf. Gleichzeitig sind die Lebensbedingungen häufig schlecht und die dort lebende Bevölkerung ist in besonderem Maße auf klimasensitive natürliche Ressourcen und ökosystemare Dienstleistungen wie auf Landwirtschaft oder Fischerei angewiesen.

Zudem stehen Entwicklungsländern kaum Kapazitäten zur Verfügung, um sich auf die Folgen des Klimawandels vorzubereiten. Der kürzlich erschienene WeltRisikoBericht des „Bündnis Entwicklung Hilft" kam zu dem Ergebnis, dass Anpassungskapazitäten ein eindeutiges Nord-Süd-Gefälle aufweisen und der Mangel an Anpassungskapazitäten oftmals ausschlaggebend für eine hohe Verletzlichkeitssituation in Entwicklungsländern ist (Birkmann/Krause et al. 2011). Auf diese Problematik hat auch schon der IPCC-Sachstandsbericht im Jahr 2001 ausdrücklich hingewiesen, wobei Anpassungskapazitäten als finanzielle Ressourcen, Technologie, Bildung, In- 
formation, Fachwissen, Infrastruktur und Leistungsvermögen im Management definiert werden (IPCC 2001). Menschen in Entwicklungsländern haben meist schlechteren Zugang zu Informationen wie Wetterwarnungen und weniger Geld- und Sachvermögen. Auch Versicherungen gegen die Folgen von extremen Wetterereignissen stehen kaum zur Verfügung. Autoritäre politische Regime erschweren den Zugang zu politischen Prozessen und auch zu staatlicher Unterstützung vor und nach Naturkatastrophen (Edenhofer/Wallacher et al. 2010).

\section{Internationale Zusammenarbeit}

Seit 1992 wird innerhalb der Vereinten Nationen (UN) über die Möglichkeiten und Pflichten der Mitgliedstaaten bei der Begrenzung des Klimawandels und seiner Folgen beraten. Grundlage der Verhandlungen und Vereinbarungen bildet die Klimarahmenkonvention (UNFCCC - United Nations Framework Convention on Climate Change). Die Forderung nach der Unterstützung der Entwicklungsländer bei der Anpassung an Klimafolgen hat früh Einzug in die internationale Klimapolitik gehalten. Die unterschiedlichen Verletzlichkeiten der Mitgliedstaaten in der Klimarahmenkonvention werden in Artikel 4 adressiert, wonach besonders anfällige Entwicklungsländer dabei unterstützt werden sollen, die durch die Anpassung an klimawandelbedingte Folgen entstehenden Kosten zu tragen. Auf nahezu allen folgenden Klimagipfeln wurde Anpassung an den Klimawandel und die Unterstützung für Entwicklungsländer adressiert, jedoch lange nicht mit Maßnahmen oder finanziellen $\mathrm{Zu}$ sagen belegt. Erst seit dem siebten Klimagipfel 2001 in Marrakesh existiert unter dem Dach der UN eine Finanzierungsarchitektur, aus der heraus auch Anpassungsprojekte in Entwicklungsländern finanziert werden können. Die entsprechenden Fonds werden von der Global Environment Facility (GEF) verwaltet, dem offiziellen Finanzierungsmechanismus der Klimarahmenkonvention.

Auf der Klimakonferenz in Cancún gelang zuletzt ein bemerkenswerter Fortschritt in der internationalen Klimaanpassungsdebatte. Während die 187 Unterzeichnerstaaten des Kyoto-Protokolls vergeblich versuchten, eine Nachfolgeregelung für die 2012 auslaufende Klimaschutzvereinbarung voranzutreiben, haben sich Industrie-, Schwellen- und Entwicklungsländer erstmals auf ein Rahmenabkommen zur Anpassung an den Klimawandel einigen können. Der Beschluss darüber erfolgte innerhalb der "Ad hoc Working Group on Long-term Cooperative Action (LCA)" und soll die umfassende Umsetzung der Anpassungsbelange aus der Klimarahmenkonvention vorantreiben. Die Staatengemeinschaft konstatiert in der Vereinbarung, dass Klimaanpassung die gleiche Priorität wie Klimaschutz habe und fordert alle Mitgliedstaaten auf, die eigene Anpassung zu stärken, Methoden zur Bestimmung von Verletzlichkeiten und auch Strategien zu deren Reduktion zu entwickeln. Dazu wird der Aufbau von Anpassungskapazitäten und widerstandsfähigen sozioökonomischen Systemen unterstrichen. Die Einrichtung eines sogenannten Anpassungs-Komitees soll zukünftig für eine grö-

\section{„Die Berücksichtigung von Ergebnissen der Anpassungsforschung in der Entwicklungszusammenarbeit kann zur Reduktion der Verletzlichkeit von Entwicklungsländern beitragen. “}

ßere Kohärenz bei der Behandlung von Anpassung im UNFCCC-Rahmen sorgen. Hier sollen Wissen und Erfahrung einzelner Länder gesammelt und Empfehlungen für den Verhandlungsprozess abgeleitet werden. Zusammensetzung und Arbeitsleitlinien der neuen Institution werden im Laufe des Jahres 2011 festgelegt. Außerdem einigte sich die Staatengemeinschaft auf eine umfangreichere Finanzierungsunterstützung für Entwicklungsländer.

\section{Anpassungsunterstützung in Entwicklungsländern}

Auch wenn die Diskussion über die deutsche Anpassungsstrategie vordergründig nationalstaatlich fokussiert und ein gutes Stück weit entkoppelt von der internationalen Zusammenarbeit und Gerechtigkeitsfragen scheint, hat die internationale Verpflichtung aus der Klimarahmenkonvention in den einschlägigen deutschen Strategiepapieren Berücksichtigung gefunden. Die deutsche Anpassungsstrategie an den Klimawandel (DAS) widmet globalen Anpassungserfordernissen ein eigenes, wenn auch abstraktes Kapitel. Konkretere Aktivitäten verspricht der Aktionsplan Anpassung (APA), den die Bundesregierung für 2011 angekündigt hat und der Anpassungsvorhaben in Deutschland aber auch internationale Aktivitäten konkretisieren will.

Die DAS wie auch die Klimarahmenkonvention betont die Bedeutung internationaler Anpassungskooperationen im Bereich Forschung und Entwicklung. Internationaler Wissenstransfer und Kooperation in der Anpassungsforschung bieten tatsächlich eine Möglichkeit Entwicklungsländer über finanzielle Hilfen und Entwicklungszusammenarbeit hinaus zu unterstützen. Bei der Forderung nach einer kooperativ gestalteten Anpassungsforschung muss nicht ausschließlich an große und explizit international ausgerichtete Förderprogramme gedacht werden. Auch Forschungsarbeiten, die zunächst auf nationale Anpassungserfordernisse zielen, können Orientierungs- und Vorbildcharakter haben, wenn dabei Kooperation und Transfer von vorneherein mitgedacht werden. Dabei wird der Nutzen der eingesetzten Gelder erhöht. Forschungstransfer und internationale Zusammenarbeit sind daher nicht nur Teil einer verantwortungsvollen und gerechten Klimapolitik, sie entsprechen auch dem Grundsatz eines effizienten Mitteleinsatzes. Ein Grundsatz, der in der öffentlich geförderten Forschung per se eine Handlungsmaxime sein muss.

Beispiele dafür finden sich innerhalb der Fördermaßnahme „KLIMZUG - Klimawandel in Regionen zukunftsfähig ge- $\rightarrow$ 
stalten“, mit der das Bundesministerium für Bildung und Forschung seit 2008 in sieben Regionen Deutschlands die Anpassung an den Klimawandel vorantreiben will. Dazu wurden Netzwerke gebildet, in denen das Know-how sämtlicher Akteure aus Wirtschaft, Politik, Wissenschaft und Gesellschaft gebündelt werden kann. Die Akteure identifizieren gemeinsam Verletzlichkeiten gegenüber dem Klimawandel und entwickeln darauf aufbauend Anpassungsstrategien. Dabei sind die gewählten Schwerpunkte und Maßnahmen der Regionen so unterschiedlich, wie die Klimaveränderungen und Klimafolgen, mit denen sie rechnen müssen.

Viele der KLIMZUG-Regionen haben frühzeitig begonnen, Kontakte zu internationalen Partnerregionen aufzubauen, die mit ähnlichen klimatischen Herausforderungen konfrontiert sind. Der regelmäßige Austausch mit anderen Regionen der Welt dient nicht nur der Wissensvermittlung ins Ausland. Gerade Regionen, die bereits stärker als die Mitte Europas von klimatischen Veränderungen betroffen sind, können Forscher und Anwender hierzulande an ihrem Wissen teilhaben lassen. Da gerade in küstennahen Gebieten hohe Folgeschäden des Klimawandels erwartet werden, bietet der Schwerpunkt Küstenschutz besonders interessante Transferansätze. Das Projekt RADOST beispielsweise, in dem Anpassungsstrategien für die deutsche Ostseeküste erarbeitet werden, kooperiert mit Ostseeanrainerstaaten und anderen küstennahen Ländern, beispielsweise in Slowenien und Marokko. Aus diesem Grund hat man auch den Rahmen der internationalen Klimaverhandlungen genutzt, um sich in sogenannten Side-Events vorzustellen und den Kontakt zu Entwicklungsländern wie Bangladesch auszubauen. Internationale Forschungskooperation wird aber nicht nur mit Entwicklungsländern erprobt: Aufgrund ähnlicher Rahmenbedingungen wurde der US-Bundesstaat Maryland als Partnerregion von nordwest2050 (Bremen-Oldenburg) ausgewählt. Beispielsweise haben die Häfen für beide Regionen eine herausragende Bedeutung. KLIMZUG-Nordhessen wiederrum pflegt mit dem österreichischen Waldviertel einen Erfahrungsaustausch auf europäischer Ebene, um das Transferpotenzial ihrer Anpassungsansätze kontinuierlich zu überprüfen. Erfahrungen zur grenzüberschreitenden Forschungskooperation, die in diesem Rahmen gemacht werden, können auch für den Forschungstransfer in Entwicklungsländer von Wert sein und wichtige Hinweise darauf geben, über welche Instrumente ein solcher Transfer am besten gelingen kann.

\section{Fazit}

Auch wenn innerhalb der Klimarahmenkonvention die finanziellen Anpassungshilfen für Entwicklungsländer in den letzten zehn Jahren sukzessive erhöht wurden, bleiben sie doch hinter dem eigentlichen Bedarf zurück. In Anbetracht der Schwierigkeiten, Geberländer zu weiteren finanziellen Zugeständnissen zu bewegen, spielt der Transfer von nationaler Anpassungsforschhung eine bedeutende Rolle. Die öffentlich geförderte Entwicklung von Methoden zur Bestimmung der regionalen Vulnerabi- lität und von Strategien zur Anpassung können nicht nur hierzulande Nutzen stiften, sondern auch für viele andere Länder verwendbar sein. Die Verfügbarkeit von anpassungsbezogenen Technologien und Wissensressourcen stellt wesentliche Anpassungskapazitäten dar und kann folglich zur Verletzlichkeitsreduktion von Entwicklungsländern beitragen. Die Einbeziehung solcher Forschungsergebnisse in die internationale Entwicklungsarbeit bietet dazu eine Möglichkeit und sollte weiter vorangetrieben werden. Dafür muss auch innerhalb national angelegter Forschungsprogramme verstärkt Sorge getragen werden.

\section{Anmerkung}

(1) Statt negativer Betroffenheit und Vulnerabilität wird auch zwischen Bruttound Nettovulnerabilität unterschieden. Siehe dazu beispielsweise Fichter et al. 2010

\section{Literatur}

Chrischilles, E. / Mahammadzadeh, M.: Betroffenheiten von Unternehmen und Kommunen durch den Klimawandel und Handlungsoptionen. In: Wirtschaftsdienst 91, 4/2011. S. 258-265.

Birkmann, J. / Krause, D. et al.: WeltRisikoBericht 2011. Bündnis Entwicklung Hilft, Berlin 2011.

Dietz, K.: Vulnerabilität und Anpassung gegenüber Klimawandel aus sozialökologischer Perspektive. In: Diskussionspapier 01/06 des Projektes „Global Governance und Klimawandel“. Berlin, 2006.

Edenhofer. O. / Wallacher. J. et al.: Global aber gerecht, Klimawandel bekämpfen, Entwicklung ermöglichen. Kurzfassung eines Reports des Potsdam-Instituts für Klimafolgenforschung und des Instituts für Gesellschaftspolitik München im Auftrag des Bischöflichen Hilfswerkes Misereor und der Münchener Rück Stiftung. München 2010.

FCCC/SBI/2009/L27: Matters relating to the least developed countries, Draft conclusions proposed by the Chair. 2009.

Fichter, K. / von Gleich, A. et al.: Theoretische Grundlagen für erfolgreiche Klimaanpassungsstrategien. nordwest2050. Bremen / Oldenburg 2010.

Intergovernmental Panel on Climate Change (IPCC): First Assessment Report, Overview Chapter. 1990.

Intergovernmental Panel on Climate Change (IPCC): Klimaänderung 2001: Auswirkungen, Anpassung und Anfälligkeit. Genf 2001.

Intergovernmental Panel on Climate Change (IPCC): Klimaänderung 2007: Synthesebericht. Berlin 2008.

United Nations Framework Convention on Climate Change (UNFCCC): Report of the Conference of the Parties on its sixteenth session, held in Cancún from 29 November to 10 December 2010. 2011.

Zebisch, M. / Grothmann, T. et al.: Klimawandel in Deutschland - Vulnerabilität und Anpassungsstrategien klimasensitiver Systeme. UBA (Umweltbundesamt). Berlin 2005.

\section{AUTORIN + KONTAKT}

Esther Chrischilles ist wissenschaftliche Mitarbeiterin am Institut der deutschen Wirtschaft Köln in der Forschungsstelle Umwelt- und Energieökonomik.

Forschungsstelle Umwelt- und Energieökonomik, Institut der deutschen Wirtschaft Köln, Postfach 101942 50459 Köln. Tel.: +49 221 4981-770, E-Mail: chrischilles@iwkoeln.de 


\section{Lizenzhinweis}

Die Beiträge in ÖkologischesWirtschaften werden unter der Creative-Commons-Lizenz "CC 4.0 Attribution Non-Commercial No Derivatives" veröffentlicht. Im Rahmen dieser Lizenz muss der Autor/Urheber stets genannt werden, das Werk darf nicht bearbeitet, abgewandelt oder in anderer Weise verändert und außerdem nicht kommerziell genutzt werden. Die digitale Version des Artikels bleibt für zwei Jahre Abonnent/innen vorbehalten und ist danach im Open Access verfügbar. 